\title{
Hypofractionated Radiotherapy as Adjuvant Treatment in Early Breast Cancer. A Review and Meta-Analysis of Randomized Controlled Trials
}

\author{
Wilfried Budach Edwin Bölke Christiane Matuschek \\ Department of Radiation Oncology, Heinrich Heine University of Düsseldorf, Germany
}

\section{Keywords}

Breast cancer - Radiotherapy · Hypofractionation · Hypofractionated · Adjuvant · Meta-analysis

\section{Summary}

Background: Adjuvant radiotherapy after breast-conserving surgery is indicated in the vast majority of breast cancer patients. Conventionally fractionated radiotherapy with $50 \mathrm{~Gy}$ in 25 fractions was considered standard of care for several decades. The recently publishes longterm results of randomized trials that have tested different moderately hypofractionated radiotherapy schedules that may change clinical practice. Patients and Methods: A Pubmed search was carried out to identify the relevant publications on hypofractionated radiotherapy in breast cancer. In total, 4 randomized controlled trials representing the results of 7,095 patients with 10 years of followup were identified. A meta-analysis on the primary end point ipsilateral breast cancer recurrence and a review of the toxicity data were performed. Results: Moderately hypofractionated radiotherapy using schedules such as $40 \mathrm{~Gy}$ in 15 fractions administered within 3 weeks are as efficient and safe as conventionally fractionated radiotherapy for most breast cancer patients who need adjuvant radiotherapy after breast-conserving surgery. In patients aged $<40$ years, after neoadjuvant chemotherapy, and if regional lymph node radiotherapy is indicated, further data are needed. Conclusion: Moderately hypofractionated radiotherapy can be recommended as standard treatment after breast-conserving surgery in the majority of breast cancer patients.

(C) 2015 S. Karger GmbH, Freiburg

\section{Introduction}

One of the important advances in the treatment of breast cancer was the replacement of mastectomy by breast-conserving surgery followed by adjuvant radiotherapy as standard treatment of early breast cancer. Large clinical trials with a follow up of 20-30 years have shown that this treatment is oncologically safe for the patients and results in good to excellent cosmetic results in approximately $80 \%$ of patients [1-4]. Most patients in these trials received conventionally fractionated radiotherapy typically consisting of 25 fractions of 2 Gy administered over 5 consecutive weeks. Hypofractionated radiotherapy by definition uses a smaller number of fractions and doses per fraction above 2 Gy. Early experiences on hypofractionated radiotherapy were mainly available from postmastectomy radiotherapy trials, and reported a high rate of quite devastating late radiation morbidity including severe fibrosis, plexopathy, and rib fractures [5-7]. In addition, experimental data indicated that most tumors, including breast cancer, exhibit a low fractionation sensitivity (high alpha/beta value) [8], whereas radiation-induced late normal tissue damage exhibits a high fractionation sensitivity (low alpha/beta value) [9]. Consequently, hypofractionated radiotherapy was thought to be harmful to patients and conventionally fractionated radiotherapy considered as standard of care in most countries $[10,11]$. However, retrospective data indicated that the use of hypofractionated radiotherapy in 13-16 fractions using 2.5-3.3 Gy per fractions to decreased total doses of $39-43$ Gy is not associated with high radiation-induced acute and late toxicity and seemed to result in local recurrence rates as low as those achieved with conventionally fractionated radiotherapy in the adjuvant setting [12-15]. These observations prompted research groups first from Ontario and later from the United Kingdom to compare adjuvant hypofractionated radiotherapy to adjuvant conventionally fractionated radiotherapy in preferentially early breast cancer. Potential benefits of hypofractionated radio- 
therapy include improved convenience for the patients due to the lower number of radiotherapy sessions, less acute skin toxicity as a result of lower total doses, and lower costs for the healthcare system.

\section{Patients and Methods}

Randomized trials on adjuvant hypofractionated radiotherapy in breast cancer were detected by performing a Pubmed search using the terms '(hypofractionated or hypofractionation or fraction size)' and '(radiotherapy or radiation or irradiation)' and 'breast cancer' limited to 'randomized controlled trial'. The search resulted in 21 hits. Considering only trials that had already published efficacy data and sufficient follow-up (> 5 years) yielded 4 trials [16-23] relevant for this review and meta-analysis of efficacy. These 4 randomized controlled trials tested different schedules of hypofractionated radiotherapy against conventionally fractionated radiotherapy (50 Gy in 25 fractions in 3-5 weeks). The expected effects of the different radiation schedules on normal tissues and tumor according to the prediction of the radiobiological linear quadratic model

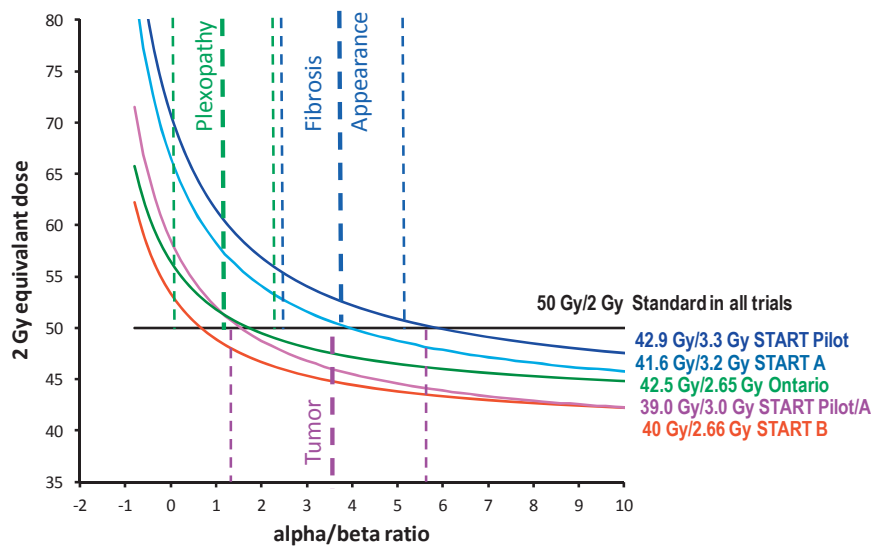

Fig. 1. Prediction of effects on tumor and normal tissues expressed as $2 \mathrm{~Gy}$ equivalent doses according to the linear quadratic model not considering repopulation. Conventional fractionation using 2 Gy per fraction (straight green line) compared to the hypofractionated radiotherapy schedules used in the different randomized trials (curved lines). are illustrated in figure 1 . The START Pilot $[17,18]$ and START A [22] trials compared conventionally fractionated radiotherapy each to 2 different 5 -week schedules of hypofractionated radiotherapy in a 1:1:1 design, whereas in the Ontario trial $[19,20]$ and the START B trial [23], 3-week hypofractionated radiation schedules were compared to conventional fractionation in a 1:1 design. A consecutive conventionally fractionated boost radiotherapy to the tumor bed was administered in none of the patients in the Ontario trial and in $43-75 \%$ in the other trials. Further details of patients and treatments are provided in table 1. Overall, the distribution of breast cancer characteristics in the trials was quite typical for patients treated with breast-conserving surgery and adjuvant radiotherapy, with the exception of the Ontario trial in which node-positive patients were excluded. About $5 \%$ of all patients received a mastectomy, and approximately $8 \%$ received regional lymph node radiation. The primary end point of all trials was local tumor recurrence in the ipsilateral breast. Cosmetic outcome was assessed by consecutive photographs and self-assessment in the START trials and by the RTOG-EORTC Late Radiation Morbidity Scoring System in the Ontario trial. At the time of the latest reports, follow-up of approximately 10 years was completed in all trials. The risk of bias in the trials has already been assessed by a Cochrane systematic review [24] and attested a low risk or unclear risk of bias for the most relevant biases, indicating an overall satisfactory quality of the conduction and reporting of the trials. At the time of the Cochrane systematic review, follow-up of the START A and B trials was only 5 years. Outcome data with 10 years of follow-up have since been published [16]. This prompted us to perform a literature-based meta-analysis using the updated hazard ratios for the primary end point (ipsilateral breast cancer recurrence) of these trials from the published material and employing a commercial software package (MIX pro ${ }^{\mathrm{TM}}$, BiostatXL, Sunnyvale, CA, USA). For all other end points, no formal meta-analysis was performed.

\section{Results}

\section{Effects of Hypofractionated Radiotherapy on Breast Cancer}

None of the trials reported a significant difference in the ipsilateral breast recurrence rate for any of the tested hypofractionated radiation schedules. However, trends towards increased local relapse rates were observed in the $39 \mathrm{~Gy}$ in 13 fractions arms of the START Pilot trial and the START A trial (cumulative hazard ratio (HR) 1.26, 95\% confidence interval (CI) 0.96-1.65; $\mathrm{p}=0.096$ ), whereas in all other hypofractionated schedules a tendency towards lower local relapse rates was detected (cumulative HR 0.86,
Table 1. Treatment and patient characteristics

\begin{tabular}{lllll}
\hline & Ontario & START Pilot & START A & START B \\
\hline Country & Canada & UK & UK & UK \\
Time of accrual & $1993-1996$ & $1986-1998$ & $1998-2002$ & $1999-2001$ \\
patients, $\mathrm{n}$ & 1234 & 1410 & 2236 & 2215 \\
Mastectomy & $0 \%$ & $0 \%$ & $15 \%$ & $8 \%$ \\
Standard-RT & $50 \mathrm{~Gy} / 25 \mathrm{fx}$ in 5 we. & $50 \mathrm{~Gy} / 25 \mathrm{fx}$ in $5 \mathrm{we}$. & $50 \mathrm{~Gy} / 25 \mathrm{fx}$ in 5 we. & $50 \mathrm{~Gy} / 25 \mathrm{fx}$ in 5 we. \\
Hypofract. RT $(1)$ & $42.5 \mathrm{~Gy} / 16 \mathrm{fx}$ in 3.1 we. & $39 \mathrm{~Gy} / 13 \mathrm{fx}$ in 5 we. & $39 \mathrm{~Gy} / 13 \mathrm{fx}$ in 5 we. & $40 \mathrm{~Gy} / 15 \mathrm{fx}$ in 3 we. \\
Hypofract. RT $(2)$ & - & $42.9 \mathrm{~Gy} / 13 \mathrm{fx}$ in $5 \mathrm{we}$. & $41.6 \mathrm{~Gy} / 13 \mathrm{fx}$ in $5 \mathrm{we}$. & - \\
Boost-RT & $0 \%$ & $74.5 \%(14 \mathrm{~Gy} / 7 \mathrm{fx})$ & $60.6 \%(10 \mathrm{~Gy} / 5 \mathrm{fx})$ & $42.6 \%(10 \mathrm{~Gy} / 5 \mathrm{fx})$ \\
Regional-RT & $0 \%$ & $20.6 \%$ & $14.2 \%$ & $7.3 \%$ \\
Mean age & $50-59$ years & $54.5 \mathrm{years}$ & $57.2 \mathrm{years}$ & 57.4 years \\
LN positive & $0 \%$ & $32.7 \%$ & $28.8 \%$ & $22.8 \%$ \\
Tumor size $>=\mathrm{T} 2$ & $20.0 \%$ & $42.5 \%$ & $48.6 \%$ & $35.9 \%$ \\
Adjuvant CHX & $11.0 \%$ & $13.9 \%$ & $35.5 \%$ & $22.2 \%$ \\
\hline
\end{tabular}

$\mathrm{RT}=$ radiotherapy; $\mathrm{fx}=$ fractions; $\mathrm{LN}=$ lymph nodes; we. = weeks; $\mathrm{CHX}=$ chemotherapy; Ontario [19, 20], START Pilot $[17,18]$, START A $[16,22]$ and B $[16,23]$ 
Table 2. Meta-analysis of randomized trials comparing hypofractionated radiotherapy to conventionally fractionated radiotherapy (fixed effects model)

\begin{tabular}{|c|c|c|c|c|c|c|c|c|c|c|}
\hline Trial & Dose, Gy & $\begin{array}{l}\text { HFX, } \\
\text { weeks }\end{array}$ & $\begin{array}{l}\text { HFX vs. CF, } \\
\mathrm{n}\end{array}$ & $\begin{array}{l}\text { Recurr } \\
\text { HFX at } \\
10 y, \%\end{array}$ & $\begin{array}{l}\text { Recurr } \\
\text { CF at } \\
10 y, \%\end{array}$ & HR & CL- & CL+ & $\mathrm{p}$ & $\begin{array}{l}\text { Weight, } \\
\%\end{array}$ \\
\hline START Pilot [17] & 42.9 vs. 50 & 5 & 466 vs. 470 & 9.6 & 12.1 & 0.86 & 0.57 & 1.30 & 0.47 & 17 \\
\hline START Pilot [17] & 39 vs. 50 & 5 & 474 vs. 470 & 14.8 & 12.1 & 1.33 & 0.92 & 1.92 & 0.13 & 21 \\
\hline START A [23] & 41.6 vs. 50 & 5 & 750 vs. 749 & 5.6 & 6.7 & 0.91 & 0.59 & 1.38 & 0.66 & 16 \\
\hline START A [23] & 39 vs. 50 & 5 & 737 vs.749 & 8.1 & 6.7 & 1.18 & 0.79 & 1.76 & 0.42 & 18 \\
\hline START B [23] & 40 vs. 50 & 3 & $\begin{array}{l}1,110 \text { vs. } \\
1,105\end{array}$ & 3.8 & 5.2 & 0.77 & 0.51 & 1.16 & 0.21 & 17 \\
\hline Ontario [19] & 42.5 vs. 50 & 3 & 622 vs. 612 & 6.2 & 6.7 & 0.92 & 0.55 & 1.52 & 0.75 & 11 \\
\hline Total & & & 7,095 & 8.4 & 8.5 & 1.00 & 0.84 & 1.18 & 0.96 & 100 \\
\hline
\end{tabular}

HFX = Hypofractionation; $\mathrm{CF}=$ conventional fractionation; Recurr = ipsilateral breast cancer recurrence; at $10 \mathrm{y}=$ after 10 -year follow-up; HR = hazard ratio; CL- = lower 95\% confidence limit of the HR; CL+= upper 95\% confidence limit of the HR.
95\% CI 0.69-1.06; $\mathrm{p}=0.163$ ), probably as a consequence of slightly higher prescribed total doses. In cumulative synthesis of the HRs of all trials using the fixed effects model, hypofractionated and conventionally fractionated adjuvant radiotherapy after breast-conserving surgery were iso-effective (HR 1.00) (table 2). The absolute ipsilateral recurrence rates varied considerably between trials ranging from 3.8 to $14.8 \%$ for hypofractionated radiotherapy and from 5.2 to $12.1 \%$ for conventionally fractionated radiotherapy (table 2). The noticeably higher ipsilateral recurrence rates in the START Pilot trial are most likely a consequence of the higher frequency of node-positive patients in this trial (33\%) in conjunction with the infrequent use of adjuvant chemotherapy (14\%), which was not unusual at the time of recruitment (1986-1998). Subgroup analyses on ipsilateral breast recurrences are available from the Ontario and the START A and B trials. In the 2010 analysis of the Ontario trial, patients with high-grade tumors had significantly less ipsilateral breast recurrences in the standard fractionation arm, whereas a reverse trend was observed for patients with low- and intermediategrade tumors. This relationship was not found in the START A and B trials and not confirmed in an updated analysis of the Ontario trial after implementation of a central pathological review in 989 of 1,234 patients [25]. Tumor size, lymph node involvement, hormone receptor status, age, adjuvant chemotherapy, and adjuvant endocrine therapy were not differently influenced by the fractionation of radiotherapy.

Overall survival was not the primary end point in any of the trials but was reported from all trials. No difference in overall survival was reported from the Ontario, START Pilot, and START A trials; however, significantly better overall survival to an absolute rate of 3.3 at 10 years was observed in the START B trial (HR 0.8, 95\% CI $0.65-0.99 ; \mathrm{p}=0.042$ ). The improved survival is probably a consequence of the significantly lower distant metastasis rate in the hypofractionated arm of this trial (absolute $+3.7 \%$ ) (HR 0 $0 \cdot 74,95 \%$ CI $0.59-0.94 ; \mathrm{p}=0.014)$. In theory, this observation could be a result of the 2-week shorter overall treatment time in the hypofractionated arm ( 3 vs. 5 weeks). However, in the Ontario trial that also used a 2-week shorter radiotherapy schedule in the hypofractionated arm, no trend toward an improved survival was seen.

\section{Effects of Hypofractionated Radiotherapy on Normal Tissues}

Data on acute skin toxicity are available from the START A and $\mathrm{B}$ trials and cumulatively analyzed in a Cochrane review [24]. Although acute skin toxicity is mild in most patients regardless of the fractionation schedule used, the risk of a more pronounced skin reaction was considerably lower in the arms with hypofractionated radiotherapy (risk ratio $0.21,95 \% \mathrm{CI} 0.07-0.64 ; \mathrm{p}=0.0067$ ). At the time of the publication of the Cochrane review, data on late radiation-induced toxicity and cosmetic results were not systematically available from the START A and B trials. Accordingly, the results of the Cochrane review that did not detect any significant difference in the overall incidence of late effects comparing the tested hypofractionated and conventionally fractionated radiation schedules have to be interpreted with caution. Since then, data on late radiation-induced effects have been published from all trials with a quite sufficient follow-up of 10 years. The results are summarized in the supplemental table (www.karger.com/?DOI=439007). $39 \mathrm{~Gy}$ in 13 fractions administered within 5 weeks was tested in the START Pilot and START A trials, and induced significantly less late toxicity in both trials for most documented late effects. $43.9 \mathrm{~Gy}$ in 13 fractions within 5 weeks in the START Pilot trial was associated with a significantly higher frequency of marked changes in breast appearance and breast induration compared to conventional fractionation. This prompted the START trialists to lower the dose to $41.6 \mathrm{~Gy}$ in 13 fractions in START A. This latter regimen did not differ in terms of any documented late effects in comparison to conventionally fractionated radiotherapy (supplemental table; $w w w . k a r g e r . c o m / ? D O I=439007)$. The same is true for the comparison of treatment arms using different end points in the Ontario trial. $40 \mathrm{~Gy}$ in 15 fractions administered as experimental arm of the START B trial proved to be significantly less toxic in terms of breast shrinkage, breast edema, and the development of teleangiectasia at 10 years after treatment (supplemental table; www.karger. com/?DOI=439007). The number of events for all other relevant late toxicities such as heart disease, rib fractures, lung toxicities, plexopathy, and development of second cancers was low in all arms of all trials, indicating no difference between hypofractionated and conventionally fractionated radiotherapy. 


\section{Discussion}

\section{General}

The results of the 4 large randomized trials comparing conventionally fractioned adjuvant radiotherapy (50 Gy/25 fractions in 5 weeks) in breast cancer to moderately hypofractionated radiotherapy with a reduction in the total dose by approximately $10 \%$ (3942.9 Gy/13-16 fractions in 3-5 weeks) indicate that hypofractionated radiotherapy can be safely used in most breast cancer patients. Fears that hypofractionated radiotherapy could result in an unacceptably high rate of late radiation-induced toxicity were not confirmed [5-7]. The follow-up of 10 years in the trials is long enough to most likely exclude considerable changes in outcomes with longer follow-up. The late toxicity in the START trials may not have been evaluated with the optimal tools, but can be regarded as sufficient to exclude that relevant toxicities were not detected.

A detailed evaluation of the results indicates that not all tested hypofractionated regimens are equally suitable for clinical use. Although 39 Gy in 13 fractions was shown to be associated with less acute and late toxicity compared to conventionally fractionated radiotherapy, one has to keep in mind that a trend towards slightly increased ipsilateral breast cancer recurrences was observed in both trials (START Pilot and START A) testing this regimen. Consequently, 39 Gy in 13 fractions should not be preferentially used. The same applies for the use of 42.9 Gy in 13 fractions, since this schedule resulted in significantly increased late toxicity. The remaining schedules, 40 Gy in 15 fractions, 42.5 Gy in 16 fractions, and 41.6 Gy in 13 fractions, are all suitable for routine clinical use; however, the most favorable observations were reported for the START B regimen. 40 Gy administered in 15 fractions of 2.67 Gy within 3 weeks resulted in significantly less acute and late toxicity and was associated with a trend towards a lower rate of ipsilateral breast recurrence. In addition, a significantly reduced distant metastasis rate and significantly improved overall survival were reported from this regimen. Although the START trialists themselves do not claim a causal relationship between the use of hypofractionation and the observation of a lower distant metastasis rate and improved survival [16], in view of no significant differences in local tumor control and no trend towards the same relationship in the similarly designed Ontario trial [19] one cannot entirely exclude a beneficial effect as the result of the substantially shorter overall radiotherapy treatment time. Since the 3-week schedule is also more convenient for most patients, the START B regimen should be the preferred schedule.

The question arises whether hypofractionated radiotherapy should be used as the preferred schedule for adjuvant radiotherapy in all breast cancer patients. Patients below 40 years, with locally advanced breast cancer, and those having undergone mastectomy were not well represented in the hypofractionation trials. Consequently, some national treatment guidelines do not recommend hypofractionated radiotherapy for these patients [26-28], whereas others do [29]. From a radiobiological point of view, it is unlikely that hypofractionated radiotherapy in younger patients or patients after mastectomy or those with locally advanced cancer would result in substantially different outcomes.

\section{Chemotherapy}

Adjuvant chemotherapy was administered in approximately 1,600 patients in the hypofractionation trials. Subgroup analyses from the START A and B [16] trials did not give any indication that hypofractionation should not be employed if adjuvant chemotherapy is given. None of the patients in the trials on hypofractionated radiotherapy received neoadjuvant chemotherapy. Whether the use of hypofractionated radiotherapy after neoadjuvant chemotherapy is safe for the patients, is formally unknown, and it is therefore not generally recommended in this situation. However, no substantial change in the fractionation sensitivity of tumors and normal tissues induced by chemotherapy was observed in both experimental and clinical data $[30,31]$ indicating that hypofractionated radiotherapy is probably also safe in this clinical setting. Further well documented clinical observations are needed to confirm the safety of hypofractionation after neoadjuvant chemotherapy.

\section{Anti-HER2 Therapy}

Trastuzumab and other drugs targeting the HER2 receptor were not clinically available during the accrual time of the trials on hypofractionated radiotherapy. Trastuzumab can be safely used after or concurrently with conventionally fractionated radiotherapy after breast-conserving surgery or mastectomy [32] at least if no radiotherapy for the treatment of the internal mammary chain lymph nodes is administered. In the palliative setting, trastuzumab was administered in combination with hypofractionated radiotherapy in different clinical situations [33-35]. No relevantly enhanced acute or late toxicity has been reported. Keeping in mind the limited long-term follow-up in the palliative setting and the lack of data with hypofractionated radiotherapy in combination with trastuzumab in the curative setting, cautious use of hypofractionated radiotherapy is appropriate before data on long-term outcome are available.

\section{Regional Radiotherapy and Cardiac Toxicity}

Radiotherapy to the supra/infraclavicular and axillary regional lymph nodes was administered in 583 patients in the hypofractionated arms of the 4 randomized trials. Shoulder stiffness and edema of the arm were not more frequently reported after hypofractionated radiotherapy compared to conventionally fractionated radiotherapy (table $3 \mathrm{a}$ and b). 1 case of brachial plexopathy was reported after 41.6 Gy in 13 fractions out of 211 treated patients in the START A trial. Keeping in mind that brachial plexopathy may manifest itself as late as 30 years after radiotherapy and that at 10 years approximately $75 \%$ of the final incidence of plexopathy is reached [6], one can calculate the worst case upper $95 \%$ confidence limits for plexopathy at 30 year after hypofractionated radiotherapy at $<6 \%$ for the START A and $<8 \%$ for the START B regimens. Although it is unlikely that these worst-case-scenario incidences will ever be observed, hypofractionated radiotherapy of the supra/ infraclavicular lymph nodes should be used with caution before further evidence is available. If used in the current situation, the 40 Gy in 15 fractions regimen should be preferred, and overdosing at the brachial plexus structures should be avoided. No long-term 
Table 3. Consensus recommendation of the AGO and DEGRO for hypofractionated adjuvant radiotherapy for invasive breast cancer (whole-breast irradiation)

\begin{tabular}{ll}
\hline$<40$ years & $\begin{array}{l}\text { conventional RT (25-28 fractions) with integrated or } \\
\text { sequential boost }\end{array}$ \\
40-65 years & $\begin{array}{l}\text { conventional RT with integrated or sequential boost, or } \\
\text { hypofractionated RT with sequential boost }\end{array}$ \\
$>65$ years & low risk: consider hypofractionated RT without boost \\
& (15-16 fractions) \\
& high risk: RT as for 40-65 years; in elderly patients individual \\
& counseling including omission of radiotherapy according to \\
& individual risk after geriatric assessment \\
& if radiotherapy of the regional lymph nodes is included, \\
Any age & conventionally fractionated RT (25-28 fractions)
\end{tabular}

$\mathrm{RT}=$ radiotherapy.

www.ago-online.de/fileadmin/downloads/leitlinien/mamma/maerz2015/en/ 2015E_13_Adjuvant_Radiotherapy.pdf

data is available on hypofractionated radiotherapy to the internal mammary chain lymph nodes. The low doses and volumes applied with typical tangential radiotherapy after breast-conserving surgery or mastectomy as used in hypofractionation trials were not associated with any evidence of more frequent heart toxicity in the hypofractionated arm of the trials. As visualized in figure 1, using the 40 Gy in 15 fractions regimen, fractionation sensitivities for heart and lungs have to be assumed equivalent to alpha/beta ratios of below 0.75 Gy to predict more harmful effects of hypofractionation compared to conventional fractionation. The current knowledge $[36,37]$ suggests higher alpha/beta ratios for heart and lungs indicating that hypofractionation is in theory save. However, this has to be substantiated by clinical data, especially since many breast cancer patients receive potentially cardiotoxic drugs such as anthracyclines and trastuzumab.

\section{Large Breasts}

Radiotherapy of large breasts $(>1,800 \mathrm{ml})$ results in a larger dose inhomogeneity compared to smaller breast. Consequently, the risk of an overdose to some parts of the breast volume is higher in large breasts. The question arises whether this overdose is more critical if hypofractionated radiotherapy is used. Models (not shown) of overdoses using the $40 \mathrm{~Gy}$ in 15 fractions regimens compared to $50 \mathrm{~Gy}$ in 25 fractions schedules predict that even for volumes that receive 1.5-times higher doses than prescribed, $40 \mathrm{~Gy}$ in 15 fractions is less critical for normal tissues as long as the fractionation sensitivity of the normal tissue can be describes with an alpha/beta ratio of $>1$ Gy. The lower $95 \%$ limit of the alpha/beta ratio for adverse effects regarding breast appearance calculated for the START A and B trials was $2.0 \mathrm{~Gy}$, indicating that large breast volume should not be an exclusion criterion for hypofractionated adjuvant radiotherapy in breast cancer. In addition, modern treatment planning including the use of intensity-modulated radiotherapy in breast cancer are able to limit the problem of overdoses in large breasts.

\section{Integrated Boost and Extreme Hypofractionation}

Hypofractionated radiotherapy with integrated simultaneous boost radiotherapy is currently being tested in randomized trials. Early results of phase II trials indicate that acute toxicity is not enhanced and that 'early late toxicity' is also low [38]. However, radiobiological models show that hypofractionation with integrated simultaneous boost could result in more late effects or less effect at the tumor depending on the tested fractionation schedule. Hypofractionated radiotherapy with integrated simultaneous boost remains experimental and should not be used outside clinical trials. The same is true for the use of extremely hypofractionated radiotherapy using only 5 fractions of 5.7-6.0 Gy to total doses of 28.5 Gy or 30 Gy in 4 weeks. These schedules were tested by the UK FAST trialists. The $5 \times 6$ Gy schedule has already been stopped because of severe late effects, whereas the results of the $5 \times 5.7$ Gy schedule are pending [39].

\section{Conclusion}

In conclusion, moderately hypofractionated radiotherapy using schedules such as $40 \mathrm{~Gy}$ in 15 fractions administered within 3 weeks has been shown to be as efficient and safe as conventionally fractionated radiotherapy for most breast cancer patients who need adjuvant radiotherapy after breast-conserving surgery. In patients younger than 40 years, after neoadjuvant chemotherapy, and if regional lymph node radiotherapy is needed, cautious use is still recommended. The AGO (German Gynecological Oncology Working Group) and the DEGRO (German Society for Radiotherapy and Oncology) recently published a consensus guideline for the use of hypofractionated radiotherapy in breast cancer, which is summarized in table 3.

\section{Supplemental Material}

Supplemental Table. Late effects

To access the supplemental table, please refer to www.karger.com/? DOI $=439007$.

\section{Disclosure Statement}

The authors have no conflict of interest to declare. 


\section{References}

1 Litiere S, Werutsky G, Fentiman IS, et al.: Breast conserving therapy versus mastectomy for stage I-II breast cancer: 20 year follow-up of the EORTC 10801 phase 3 randomised trial. Lancet Oncol 2012;13:412-419.

2 Fisher B, Anderson S, Bryant J, et al.: Twenty-year follow-up of a randomized trial comparing total mastectomy, lumpectomy, and lumpectomy plus irradiation for the treatment of invasive breast cancer. N Engl J Med 2002;347:1233-1241.

3 Jacobson JA, Danforth DN, Cowan KH, et al.: Tenyear results of a comparison of conservation with mastectomy in the treatment of stage I and II breast cancer. N Engl J Med 1995;332:907-911.

4 Darby S, McGale P, Correa C, et al.; Early Breast Can cer Trialists' Collaborative Group: Effect of radiotherapy after breast-conserving surgery on 10-year recurrence and 15-year breast cancer death: meta-analysis of individual patient data for 10,801 women in $17 \mathrm{ran}$ domised trials. Lancet 2011;378:1707-1716.

5 Overgaard M, Bentzen SM, Christensen JJ, Madsen $\mathrm{EH}$ : The value of the NSD formula in equation of acute and late radiation complications in normal tissue following 2 and 5 fractions per week in breast cancer patients treated with postmastectomy irradiation. Radiother Oncol 1987;9:1-11.

6 Galecki J, Hicer-Grzenkowicz J, Grudzien-Kowalska $\mathrm{M}$, et al.: Radiation-induced brachial plexopathy and hypofractionated regimens in adjuvant irradiation of patients with breast cancer - a review. Acta Oncol 2006; 45:280-284

7 Overgaard M: Spontaneous radiation-induced rib fractures in breast cancer patients treated with postmastectomy irradiation. A clinical radiobiological analysis of the influence of fraction size and dose-response relationships on late bone damage. Acta Oncol 1988;27: 117-122.

8 Stuschke M, Budach V, Sack H: Radioresponsivenes of human glioma, sarcoma, and breast cancer spheroids depends on tumor differentiation. Int J Radiat Oncol Biol Phys 1993;27:627-636.

9 Van Limbergen E, Rijnders A, van der Schueren E, et al.: Cosmetic evaluation of breast conserving treatment for mammary cancer. 2. A quantitative analysis of the influence of radiation dose, fractionation schedules and surgical treatment techniques on cosmetic results. Radiother Oncol 1989;16:253-267.

10 Yamada Y, Ackerman I, Franssen E, et al.: Does the dose fractionation schedule influence local control of adjuvant radiotherapy for early stage breast cancer? Int J Radiat Oncol Biol Phys 1999;44:99-104.

11 Fletcher GH: Hypofractionation: lessons from complications. Radiother Oncol 1991;20:10-15.

12 Clark RM, Whelan T, Levine M, et al.: Randomized clinical trial of breast irradiation following lumpectomy and axillary dissection for node-negative breas cancer: an update. Ontario Clinical Oncology Group. Natl Cancer Inst 1996;88:1659-1664.
13 Ash DV, Benson EA, Sainsbury JR, et al.: Seven-year follow-up on 334 patients treated by breast conserving surgery and short course radical postoperative radiotherapy: a report of the Yorkshire Breast Cancer Group. Clin Oncol (R Coll Radiol) 1995;7:93-96.

14 Olivotto IA, Weir LM, et al.: Late cosmetic results of short fractionation for breast conservation. Radiother Oncol 1996;41:7-13.

15 Shelley W, Brundage M, Hayter C, et al.: A shorter fractionation schedule for postlumpectomy breast cancer patients. Int J Radiat Oncol Biol Phys 2000;47: 1219-1228.

16 Haviland JS, Owen JR, Dewar JA, et al.: The UK Standardisation of Breast Radiotherapy (START) trials of radiotherapy hypofractionation for treatment of early breast cancer: 10-year follow-up results of two randomised controlled trials. Lancet Oncol 2013;14:10861094

17 Owen JR, Ashton A, Bliss JM, et al.: Effect of radiotherapy fraction size on tumour control in patients with early-stage breast cancer after local tumour excision: long-term results of a randomised trial. Lancet Oncol 2006;7:467-471.

18 Yarnold J, Ashton A, Bliss J, et al.: Fractionation sensitivity and dose response of late adverse effects in the breast after radiotherapy for early breast cancer: longterm results of a randomised trial. Radiother Oncol 2005;75:9-17.

19 Whelan TJ, Pignol JP, Levine MN, et al.: Long-term results of hypofractionated radiation therapy for breast cancer. N Engl J Med 2010;362:513-520.

20 Whelan T, MacKenzie R, Julian J, et al.: Randomized trial of breast irradiation schedules after lumpectomy for women with lymph node-negative breast cancer. J Natl Cancer Inst 2002;94:1143-1150.

21 Yarnold J, Bentzen SM, Coles C, Haviland J: Hypofractionated whole-breast radiotherapy for women with early breast cancer: myths and realities. Int J Radiat Oncol Biol Phys 2011;79:1-9.

22 Group ST, Bentzen SM, Agrawal RK, et al.: The UK Standardisation of Breast Radiotherapy (START) Trial A of radiotherapy hypofractionation for treatment of early breast cancer: a randomised trial. Lancet Oncol 2008;9:331-341.

23 Group ST, Bentzen SM, Agrawal RK, et al.: The UK Standardisation of Breast Radiotherapy (START) Trial $B$ of radiotherapy hypofractionation for treatment of early breast cancer: a randomised trial. Lancet 2008; 371:1098-1107.

24 James ML, Lehman M, Hider PN, et al.: Fraction size in radiation treatment for breast conservation in early breast cancer. Cochrane Database Syst Rev 2010;(11): CD003860.

25 Bane AL, Whelan TJ, Pond GR, et al.: Tumor factors predictive of response to hypofractionated radiotherapy in a randomized trial following breast conserving therapy. Ann Oncol 2014;25:992-998.
26 Sedlmayer F, Sautter-Bihl ML, Budach W, et al. DEGRO practical guidelines: radiotherapy of breast cancer I: radiotherapy following breast conserving therapy for invasive breast cancer. Strahlenther Onkol 2013;189:825-833.

27 Senkus E, Kyriakides S, Penault-Llorca F, et al.: Primary breast cancer: ESMO Clinical Practice Guidelines for diagnosis, treatment and follow-up. Ann Oncol 2013;24(suppl 6):vi7-23.

28 Besnard S, Cutuli B, Fourquet A, et al.; French National Cancer Institute: (Radiotherapy of invasive breast cancer: French national guidelines). Cancer Radiother 2012;16:503-513.

29 Breast cancer Dutch Guideline, version 2.0. www.oncoline.nl/uploaded/docs/mammacarcinoom/Dutch\%20 Breast\%20Cancer\%20Guideline\%202012.pdf.

30 Van Rongen E, Kuijpers WC, Baten-Wittwer A: The influence of platinum drugs on the radiation response of rat kidneys. Radiother Oncol 1994;31:138-150.

31 Collette S, Collette L, Budiharto T, et al.: Predictors of the risk of fibrosis at 10 years after breast conserving therapy for early breast cancer: a study based on the EORTC Trial 22881-10882 'boost versus no boost'. Eur J Cancer 2008;44:2587-2599.

32 Halyard MY, Pisansky TM, Dueck AC, et al.: Radiotherapy and adjuvant trastuzumab in operable breast cancer: tolerability and adverse event data from the NCCTG Phase III Trial N9831. J Clin Oncol 2009;27: 2638-2644.

33 Farolfi A: The impact of radiotherapy, trastuzumab and hormonal therapy on cardiac fibrosis. More is worse? Anadolu Kardiyol Derg 2014;14:334-335.

34 Riemer AB, Zielinski CC: (Use of trastuzumab in the therapy of breast cancer). Ther Umsch 2008;65:217222

35 Jeyakumar A, Younis T: Trastuzumab for HER2-positive metastatic breast cancer: clinical and economic considerations. Clin Med Insights Oncol 2012;6:179 187.

36 Gagliardi G, Constine LS, Moiseenko V, et al.: Radiation dose-volume effects in the heart. Int J Radiat Oncol Biol Phys 2010;76(3 suppl):S77-85.

37 Marks LB, Bentzen SM, Deasy JO, et al.: Radiation dose-volume effects in the lung. Int J Radiat Oncol Biol Phys 2010;76(3 suppl):S70-76.

38 Dellas K, Vonthein R, Zimmer J, et al.; ARO Study Group: Hypofractionation with simultaneous integrated boost for early breast cancer: results of the German multicenter phase II trial (ARO-2010-01). Strahlenther Onkol 2014;190:646-653.

39 Group FT, Agrawal RK, Alhasso A, et al.: First results of the randomised UK FAST Trial of radiotherapy hypofractionation for treatment of early breast cancer (CRUKE/04/015). Radiother Oncol 2011;100:93-100. 